\title{
Robust Road Marking Extraction in Urban Environments Using Stereo Images
}

\author{
Yazid Sebsadji, Jean-Philippe Tarel, Philippe Foucher and Pierre Charbonnier
}

\begin{abstract}
Most road marking detection systems use image processing to extract potential marking elements in their first stage. Hence, the performances of extraction algorithms clearly impact the result of the whole process. In this paper, we address the problem of extracting road markings in high resolution environment images taken by inspection vehicles in a urban context. This situation is challenging since large special markings, such as crosswalks, zebras or pictographs must be detected as well as lane markings. Moreover, urban images feature many white elements that might lure the extraction process. In prior work an efficient extraction process, called Median Local Threshold algorithm, was proposed that can handle all kinds of road markings. This extraction algorithm is here improved and compared to other extraction algorithms. An experimental study performed on a database of images with ground-truth shows that the stereovision strategy reduces the number of false alarms without significant loss of true detection.
\end{abstract}

\section{INTRODUCTION}

The applications of road markings detection concern many vision-based driver assistance systems. Over the last two decades, many research teams have been interested in this topic and most of them have focused on road lane markings detection. In the context of the iTOWNS ${ }^{1}$ project, our research is interested in detecting road markings in images of complex urban scenes. This includes road lane markings as well as specific markings such as crosswalks, zebras, arrows, or pictographs. Road marking detection algorithms are usually comprised of three stages :

- extraction of marking elements,

- geometrical model estimation,

- tracking and filtering of the parameters of the geometrical model along an image sequence.

Of course, the efficiency of the first stage has a strong impact on the overall performance of the system. The main objective of this paper is to evaluate different extraction algorithms, with regard to both lane detection and specific markings detection, in the spirit of [18], but considering special markings, and not only lane markings. A second

This work is partly funded by the ANR (French National Research Agency) within iTowns-MDCO project.

Yazid Sebsadji, Philippe Foucher and Pierre Charbonnier are with LRPC Strasbourg (ERA 27 LCPC), 11 Rue Jean Mentelin, BP 9, F-67200 Strasbourg, France, \{yazid.sebsadji, philippe.foucher, pierre.charbonnier\}

@developpement-durable.gouv.fr

Jean-Philippe Tarel is with Univ. Paris-Est, INRETS-LCPC, $58 \mathrm{Bd} \mathrm{Le}$ febvre, F-75015 Paris, France, jean-philippe.tarel@lcpc.fr

$1_{\text {WWW. }}$ itowns. fr contribution aims at proposing stereo versions of our extractors. The goal of stereo selection is to reduce the number of false alarms which may be fairly high in urban environment. Finally, we compare the performances of the single-image and stereo algorithms.

Almost all extraction methods rely on the fact that road markings are standardized objects, namely bands painted on the road whose width and color are fixed by technical norms and standards. In Europe, road markings are most of the time white and more occasionally green, yellow or blue. Different approaches have been proposed to extract road markings from images, we refer the reader to [9] for a global survey. In [1], the extraction is performed on the Inverse Perspective Mapping (IPM) of the input color image using a Gaussian filter that detects vertical strips. A similar approach is proposed by [3] in the case of night-time road lane detection. In [18], six extractors have been systematically evaluated on a database of 116 high-resolution images called ROMA (www.lcpc.fr/en/produits/ride/). The best performances were obtained by local threshold extractors. These algorithm are described in Sect. III, where more efficient variants are also proposed. Except [18], the extraction step is seldom assessed independently and few authors propose a quantitative evaluation of the global road marking detection system [9], [8].

It may be noticed that the authors rarely consider both special markings and road lane markings. Most of the studies on specific marking detection concern pedestrian crossings. The detection methods are then based either on the Hough transform [13], or on graphical models [4] or on projective invariants [17]. To our knowledge, very few researches have concerned other kinds of special road markings (e.g. pictograms, text, arrows, zebras). We just mention [6] which proposes to extract and recognize road arrows by regionbased segmentation, connected component extraction and geometric selection. In the context of lane detection, stereovision versions of markings extractors have been proposed in [7], [6], [10], [2] and for rectangular road markings and crosswalks, in [14].

The paper is organized as follows. We first describe, in Sect. II, our experimental setup. Then, Sect. III introduces our single image extraction algorithms, which are extended to stereovision in Sect. IV. In Sect. V, single and stereo results are compared and discussed. Finally, Sect. VI provides conclusions and perspectives. 


\section{EVALUATION DATA SET, GROUND TRUTH LABELING AND EVALUATION METRICS}

\section{A. Evaluation Data set}

The database we use in this paper is called MiTowns. It comprises 47 pairs of images of size $1920 \times 1080$. The images have been captured in urban area (Paris, France) by two stereoscopic cameras mounted on an inspection vehicle of the French Institut Géographique National IGN. For each image, the results of the extraction algorithms are compared to the corresponding ground truth image, in which road marks have been manually selected. In the ground truth images, a different label is set for each kind of marks.

The database represents a sampling of possible road markings : lane markings, crosswalks, arrows, bus lane markings, bicycle lane markings (see Fig. 8, $1^{\text {st }}$ column). Complex situations (occluded, worn-out, dirty markings, variations in road material) and variable lighting conditions (shadows, bright sun) are also considered in the images selection to build a difficult database. We note that in the iTowns project, the images acquisition is done only during the day (not during the night).

\section{B. Evaluation metrics}

To optimize and evaluate the performances of each extraction algorithm, we use two classical tools : Receiver Operating Characteristic (ROC) curves [5] and Dice Similarity Coefficient (DSC, also named F-measure).

ROC curves plot the True Positive Rate (TPR) versus the False Positive Rate (FPR). Each point of the curve corresponds to a particular setting of the extractor parameters. In this paper, all ROC curves are plotted by varying the detection threshold $T_{G}$ (see Sec. III) over the range $[0 ; 255]$. TPR and FPR are defined as :

$$
T P R=\frac{T P}{P} \quad F P R=\frac{F P}{N}
$$

where $P$ and $N$ are respectively the numbers of positives and negatives in the Ground Truth images, $T P \leq P$ is the number of True Positives and $F P \leq N$ is the number of false positives.

When a ROC curve is always above another one, the algorithm associated with the first ROC curve is better. The situation is less clear when the two ROC curves cross each other. Since the proportion of pixels corresponding to markings in the image is small, our ROC curve analysis will focus on low False Positive Rates (left part of the curve). Therefore, the Dice Coefficient Similarity (DSC) is used in complement [18]. The DSC is defined as :

$$
D S C=\frac{2 T P}{T P+F P+P}
$$

Plots of the Dice vs. the value of $T_{G}$ are shown in the next sections. The maximum value of the Dice is of importance to compare the different extractors, since it corresponds in some way to an optimal value of the threshold $T_{G}$. The width of the peak also informs on the sensitivity of the extractor with respect to the threshold tuning.
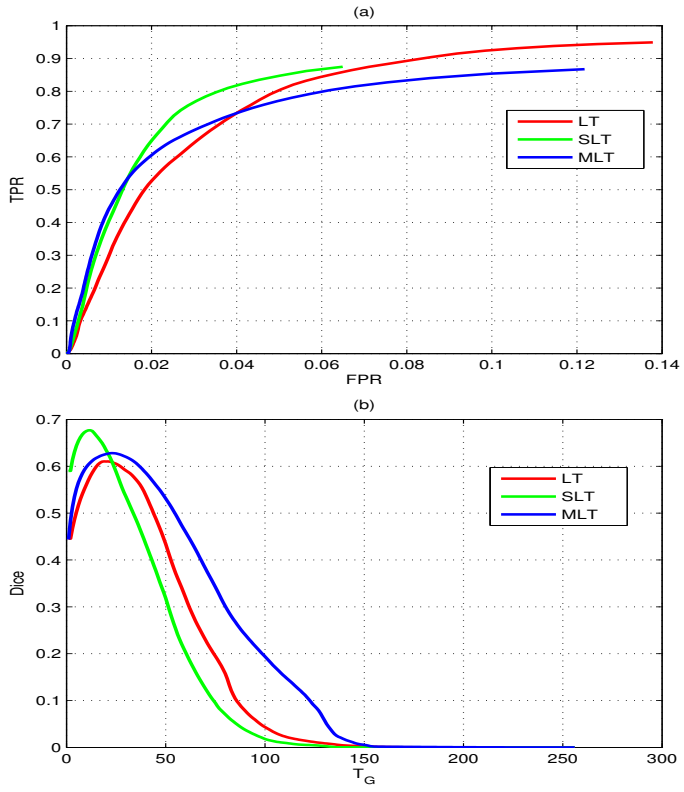

Fig. 1. Comparison between the LT, SLT and MLT $\left(50^{\text {th }}\right.$ percentile $)$ extractors : Roc curves (a), Dice curves (b).

\section{LANE MARKING EXTRACTION USING LOCAL THRESHOLD ALGORITHMS}

In most algorithms, the extraction stage processes image lines independently, in a sequential fashion, seeking segments that might correspond to lane marking elements. Road markings are white (more rarely, colored, but most of the time light) strips of constant normalized width, painted on the dark roadway. Hence, extraction algorithms exploit, more or less fully, the characteristics of road markings with respect to photometry, geometry or both. To make use of the contrast between markings and pavement, a detection threshold, $T_{G}$, is put on the intensity. From a geometric point of view, the constant width assumption is often violated due to wear, dirt and occlusions. Hence, instead of a threshold, an acceptability range is defined. Since markings are observed in perspective, this range must be adapted to the vertical position in the image (i.e. the line number, $v$ ). We will then denote it by $\left[S_{m}(v), S_{M}(v)\right]$. In the following evaluation on the Mitowns database, these parameters are fixed to same values for all the extractors. $S_{m}$ is linearly interpolated with value 1 at the line of horizon and with value 35 at the bottom of the image. To extract all the large markings, $S_{M}$ is linearly interpolated with value 1 at the line of horizon and with value 350 at the bottom of the image. These value were obtained by testing different values and by choosing the ones maximizing the DSC.

Many extractors were proposed in the literature, among which we compared in [18] the 6 most representative on a set of 116 high-resolution images named ROMA database available at www.lcpc.fr/en/produits/ride/. The best overall performance on this particular test set were obtained by Local Threshold (LT) and Symmetrical Local Threshold (SLT) algorithms, which are now presented. In the Local 
Threshold algorithm, pixels whose intensity $I(u, v)$ is higher than $T_{G}+\bar{I}(u, v)$ are selected, where $\bar{I}(u, v)$ is the intensity average over an horizontal interval of width $12 S_{M}(v)$ centered on $(u, v)$. Then, sets of connected pixels whose width is larger than $S_{m}(v)$ are extracted (i.e. selected as marking elements). The Symmetrical Local Threshold algorithm is similar, except that two average values are computed : $\bar{I}_{\text {left }}$ is the mean image intensity over the interval $\left.] u-6 S_{M}(v), u\right]$, while $\bar{I}_{\text {right }}$ is computed on $\left.] u, u+6 S_{M}(v)\right]$. Selected pixels must have higher intensity than both $T_{G}+\bar{I}_{\text {left }}$ and $T_{G}+\bar{I}_{\text {right }}$. The factor 6 and 12 in the intervals were obtained by testing different values and by choosing the ones maximizing the ROC curve on the ROMA database.

The idea behind these algorithms is that a point of marking is surrounded by pixels belonging to the pavement, thus substantially darker. Ideally, the mean value(s) should correspond to the representative gray level of the pavement around the marking strip. Unfortunately, the presence of other points of the marking, or pixels belonging to adjacent markings, biases the estimated average towards high values. It was shown in [11] that an efficient strategy was to use the median instead of the mean to better estimate the representative gray level of the pavement. The resulting algorithm will be called Median Local Threshold (MLT) hereafter. Since it can bear up to $50 \%$ erroneous data, the median is computed over an interval of width $2 S_{M}(v)$ around position $(u, v)$. Inspired by [12], a fast version of the median filtering along lines has been developped. Its complexity is a linear function of the number of pixels in the image and does not depend on the filter size. This leads to an average MLT processing time of $80 \mathrm{~ms}$ for an image of size $960 \times 540$ on a dual-core processor.

However, this strategy does not work in certain cases, especially for markings such as crosswalks or zebras : it may indeed appear that there is more than $50 \%$ white in the interval where the median is computed. This effect is particularly visible on the MiTowns database, which comprises many special markings, see Fig. 1 where the performances of the LT, SLT and MLT extractors are compared.

It is then convenient to consider a lower quantile than 0.5. To set the best value for the quantile, a series of systematic tests was performed over the MiTowns database (samples of ROC and Dice curves are shown on Fig. 2). The best result is obtained for the $43^{\text {rd }}$ percentile. Compared to the standard median $\left(50^{\text {th }}\right.$ percentile), the Dice coefficient is improved by $1.8 \%$ and the number of True Positives (TP) is improved by $17.32 \%$. An example of this improvement is given in Fig. 3 where the results obtained by the MLT with the $50^{\text {th }}$ and $43^{\text {rd }}$ percentile are compared. We will therefore use the $43^{\text {rd }}$ percentile from now on in this paper.

Finally, we illustrate in Fig. 4 the results of the LT, SLT and MLT $\left(43^{\text {rd }}\right.$ percentile) on a test image. In this example the detection threshold was optimized for each extractor. There is a clear improvement of the MLT and SLT over the LT. The difference between SLT and MLT is visually less obvious. However, we found that the best True Positive Rate is $65 \%$ for LT, $82 \%$ for SLT and $85 \%$ for MLT for this
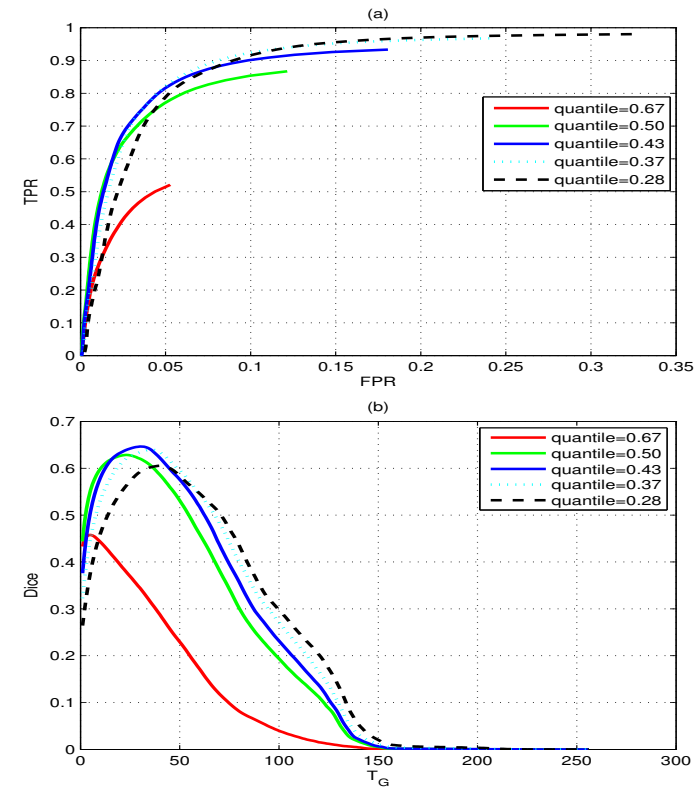

Fig. 2. Roc and Dice curves obtained for different percentiles : Roc curves (a), Dice curves (b). The best Dice is obtained for the $43^{\text {rd }}$ percentile.

particular image.

\section{MARKING EXTRACTION IN STEREO}

By comparing the results obtained on the MiTowns image database to those of [18], it might be noticed that the number of false positives is higher here than using the ROMA database. A possible explanation is that ROMA is comprised of rural images while, in this paper, we consider urban hence much more cluttered - images. Decreasing the number of false alarms is of a great interest, especially in the urban context. To this end, we shall exploit the truism that markings are painted on the road plane which, when translated into the stereovision formalism, provides an extra selection rule. This rule can be applied to the binary maps resulting from the analysis of both the left and right images of the stereo pair using a single image extraction algorithm.

\section{A. Stereo selection}

The stereo selection algorithms that will be presented now rely on the knowledge of the position and orientation of the road surface. This information can be obtained either off-line or on-line :

- Off-line : The cameras inboard the vehicle are calibrated in near field with respect to the road plane before acquisition. Roll and pitch angles only vary slightly along the vehicle trajectory due to curves, accelerations and decelerations. Thus the calibration provides an approximated road plane location for all frames.

- On-line : For each frame, the road surface can be estimated by stereovision, see for instance [15].

Of course, the uncertainty on the road surface is larger with the off-line approach than with the on-line one. We here use the off-line method to test the stereo selection in the most challenging conditions. 

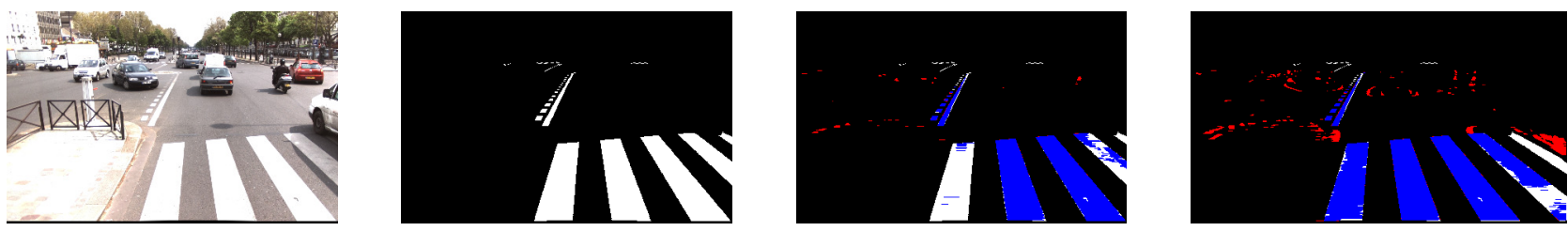

Fig. 3. (From left to right) original image, ground truth image (white : marking elements, black : non-marking pixels), extraction results of the MLT algorithm ( $50^{\text {th }}$ percentile), extraction results of the MLT algorithm ( $43^{\text {rd }}$ percentile). Red : False Positives, blue : True Positives.
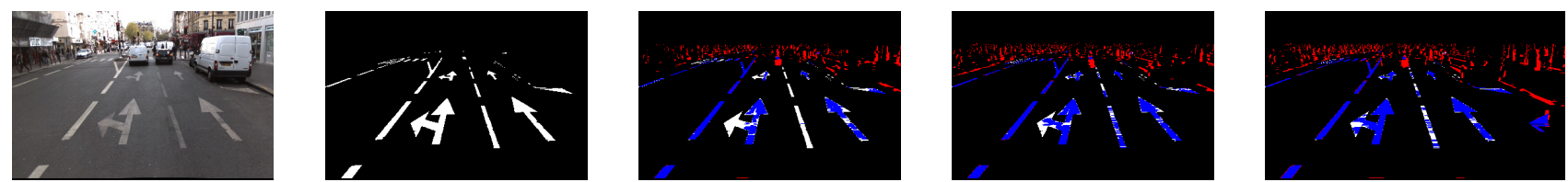

Fig. 4. (From left to right) original image, ground truth image (white : marking elements, black : non-marking pixels), extraction results of the LT algorithm, extraction results of the SLT algorithm and extraction results of the MLT algorithm ( $43^{\text {rd }}$ percentile). Red : False Positives, blue : True Positives.

For a simpler presentation, we now assume without that the left and right images are in rectified stereoscopic geometry (the general case can be also handled using epipolar geometry) and that the road is planar. Let $(u, v)$ denote the coordinate of a point of the road plane in the left view and let $\left(u^{\prime}, v^{\prime}\right)$ denote its coordinates in the right image. It is easy to show [15], that the mapping between views can be parametrized as :

$$
\left\{\begin{array}{llc}
u^{\prime} & = & u+a_{0}+a_{1} v \\
v^{\prime} & = & v
\end{array}\right.
$$

where the vertical intercept, $a_{0}$, and slope, $a_{1}$, are related to the intrinsic cameras parameters as well as to the position and orientation of the road plane. Note that this model may be extended to a polynomial relationship to tackle longitudinal variations of the road and that an additional term may be introduced to account for roll angle variations, see [15] for details. The parameters of (1) can be estimated using, for example, the method proposed in [15]. However, in this paper we use the fixed values estimated off-line by calibration.

In practice, conditions (1) are never exactly satisfied, due to many factors of error. Most of them, such as the dynamics of the vehicle, are very difficult to quantify. Hence, we propose to consider a model of the uncertainty on $u^{\prime}$ and to evaluate its parameters experimentally. Since pitch angle variations are, from our experience, predominant, the uncertainty on $u^{\prime}$ is modeled as a linear function of the vertical image coordinate only :

$$
\delta u^{\prime}(v)=b_{0}+b_{1} v
$$

The uncertainty $\delta u^{\prime}$ is thus parametrized by the intercept $b_{0}$ and the slope, $b_{1}$.

Using the mapping (1) of the road points between the left and right images and the uncertainty model (2), two algorithms are proposed to perform stereo selection :

- Pixel selection: Scanning the left extraction map, which is a binary image of the extracted markings on the left image, every marking pixel is tested. Denoting a marking pixel by $(u, v)$, a corresponding marking pixel is searched in the right image in the range $\left(u^{\prime} \pm \delta u^{\prime}(v), v\right)$. If this search succeeds, the pixel is kept as extracted in the left extraction map, while it is removed otherwise.

- Border selection : Scanning the left extraction map, every pairs of left and right borders on a line is tested. Denoting the left border by $\left(u_{l}, v\right)$ and the right border by $\left(u_{r}, v\right)$, corresponding left and right borders are searched in the right image in the location range $\left(u_{l}^{\prime} \pm \delta u^{\prime}(v), v\right)$ and $\left(u_{r}^{\prime} \pm \delta u^{\prime}(v), v\right)$, respectively. When this search succeeds, the pixels between left and right borders are kept as extracted in the left extraction map; they are removed otherwise.

Note that, the symmetrical test (with respect to left and right images) may also be performed to analyze the right extraction map instead of the left extraction map. Also note that, in terms of computational cost, the border selection algorithm is faster than the pixel selection method. However, the extra cost due to the stereo selection is quite small : $2 \mathrm{~ms}$ for the border selection and $4 \mathrm{~ms}$ for the pixel selection in average for an image of size $960 \times 540$ on a dual-core processor. Notice that the stereo selection applies only in the field of view which is common between the two views. Therefore, for the part of the image which is not in common field of view, extracted pixels are kept without selection.

\section{B. Comparison between pixel and border stereo selection}

A correct choice of the values $\left(b_{0}, b_{1}\right)$ of the uncertainty model is necessary to obtain efficient stereo selection. On the ground-truth database, we computed the ROC curves for different pairs of parameters $\left(b_{0}, b_{1}\right)$ (see Fig. 5). Samples of the obtained curves are shown for the pixel and border based stereo selections. For pixel based stereo selection, the best dice 0.699 is obtained for $b_{0}=1$ and $b_{1}=0.1$. For border based stereo selection, the best dice 0.608 is obtained for $b_{0}=5$ and $b_{1}=0.2$. These results were obtained with marking extraction using median local threshold (MLT) 

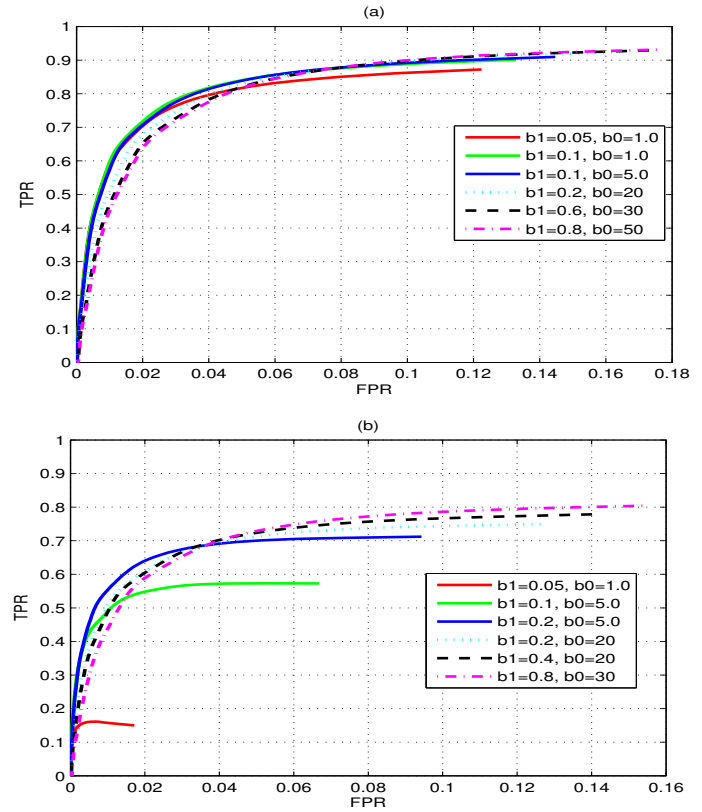

Fig. 5. ROC curves obtained for different pairs of parameters $\left(b_{0}, b_{1}\right)$ for the pixel based stereo selection (a) and for border based stereo selection (b), using MLT algorithm with $43^{\text {rd }}$ percentile.

based on $43^{\text {rd }}$-percentile, and the same optimal uncertainty parameters where obtained for other extraction algorithms. As a consequence, pixel based stereo selection is shown to be a little more efficient in terms of ROC curves and Dice coefficient than border based stereo selection (see Fig. 6). For illustration, Figure 7 shows results obtained with pixel and border stereo selections.

\section{EXPERIMENTAL RESULTS}

A double analysis of the results may be proposed. A study of the intrinsic performances of each extractor shows the maximum DSC value is obtained for SLT extractor. The TPR obtained for the optimal values $T_{G}$ of each single-image extractor are not very high : $74 \%$ for SLT, $77 \%$ for LT and $69 \%$ for MLT. These values of TPR can be explained by the difficulty of the chosen MiTowns images with respect to the task of markings extraction. We also tested the MLT extraction algorithm with $43^{\text {rd }}$ percentile on the ROMA image database and the results are very close to the one obtained using $50^{\text {th }}$ percentile MLT algorithm. The fact that the MLT algorithm better performs on the ROMA database and that it is the SLT algorithm which better performs on the MiTowns image database is interesting. It is probably explained by the difference in nature of the images, ROMA database contains rural images with mostly lane markings and MiTowns database contains urban images with many special markings. This also indicates that it is necessary to be careful in our conclusions. As a consequence, to select an extractor for a specific kind of applications, it is always requested to run an experiment to find the best one in the set of the better extractors.

A second point of view consists in considering the extractor as a first step of a marking detection algorithm in
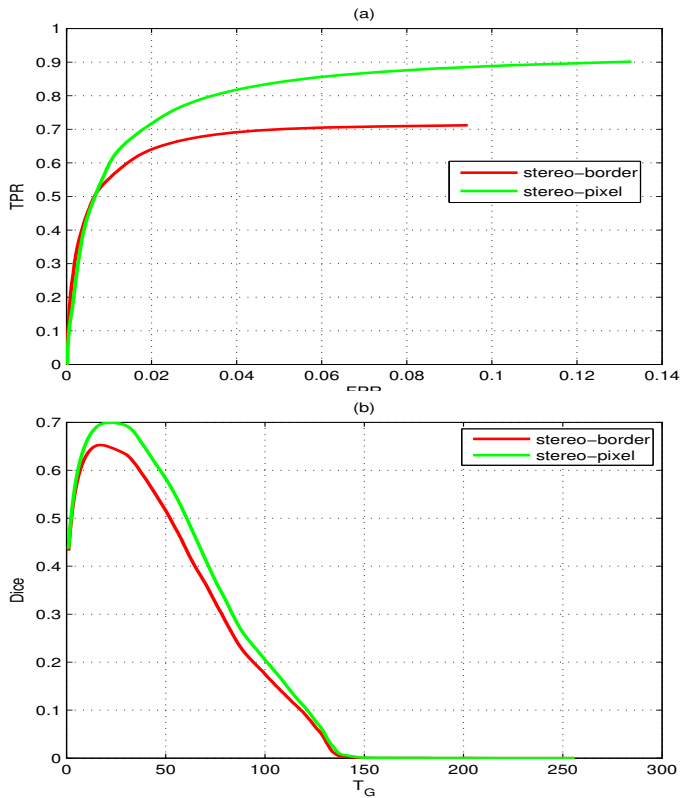

Fig. 6. Comparison of the results obtained by pixel based stereo selection and by border based stereo selection algorithms : (ROC curves (a), Dice curves (b))

which the connected component may be considered. In this case, what is important is to detect the object and to have enough pixels for a correct recognition. Thus, the impact of lost pixels when distributed around the edges is of reduced importance.

Figure 8 presents some examples of road marking extraction from the MiTowns database using the MLT algorithm with $43^{\text {rd }}$ percentile. Looking at these images, the extractor is robust to extract all kind of road markings as road lane markings (Fig 8-rows 1, 5 and 6), pedestrian crossings (Fig 8rows 2 and 3), drawings (Fig 8-row 3), zebras (Fig 8-row 4), directional arrows (Fig 8-row 5) and texts (Fig 8-row 6). Moreover, it may be noticed that partly erased road markings are also extracted as shown on Fig 8-row 1. We observe that non-detected road markings are often located on the top half of the image (Fig 8-row 4) where the low resolution of the marking (due to perspective effect) increases the difficulty of extraction task. Other missed pixels correspond to dirty or highly erased signs (for example, see the non-detected arrow on Fig 8-row 4).

The performances of the single-image and stereo version of the three extractors LT, SLT and MLT are illustrated by the ROC and DSC curves plotted on Fig. 9-a and Fig. 9-b.

Concerning the extra stereo selection, the ROC curves show that the stereo selection reduces the number of false alarms compared to the corresponding single-image algorithm, but it also slightly reduces the number of true detection. As shown in Fig 9, for the optimal values $T_{G}$, the stereo versions of MLT extractor (resp. SLT and LT extractors) enables to eliminate $47.34 \%$ (resp. $36.71 \%$ and $40.73 \%$ ) of false alarms but $6.88 \%$ (resp. $12.95 \%$ and $9.07 \%$ ) of true detections are also lost. Considering the Dice curves, the improvement of performances with stereo selection is 

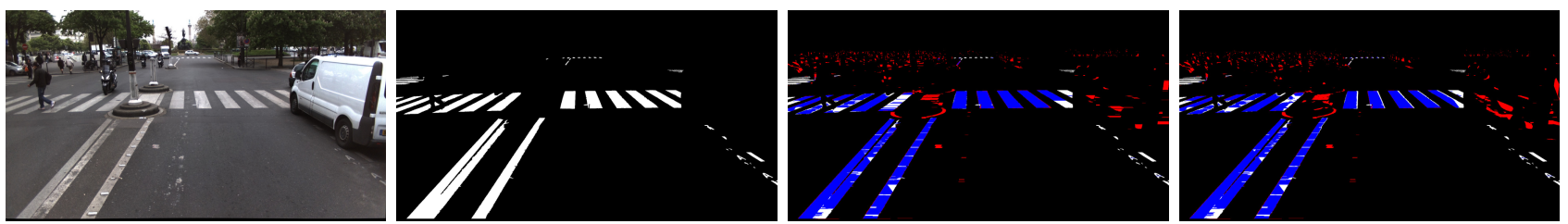

Fig. 7. (From left to right) original image, ground truth image (white : marking elements, black : non-marking pixels), result with border stereo selection, result with pixel stereo selection. Red : False Positives, blue : True Positives.
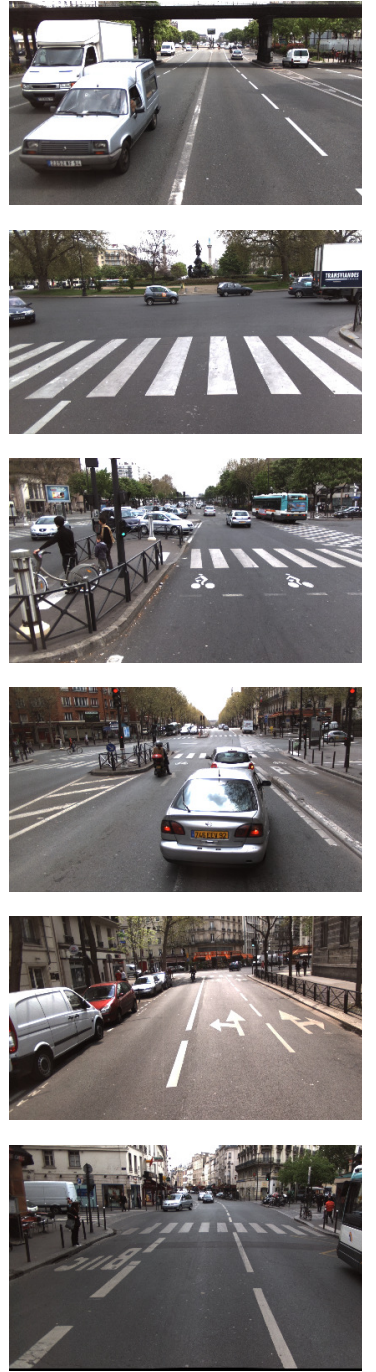
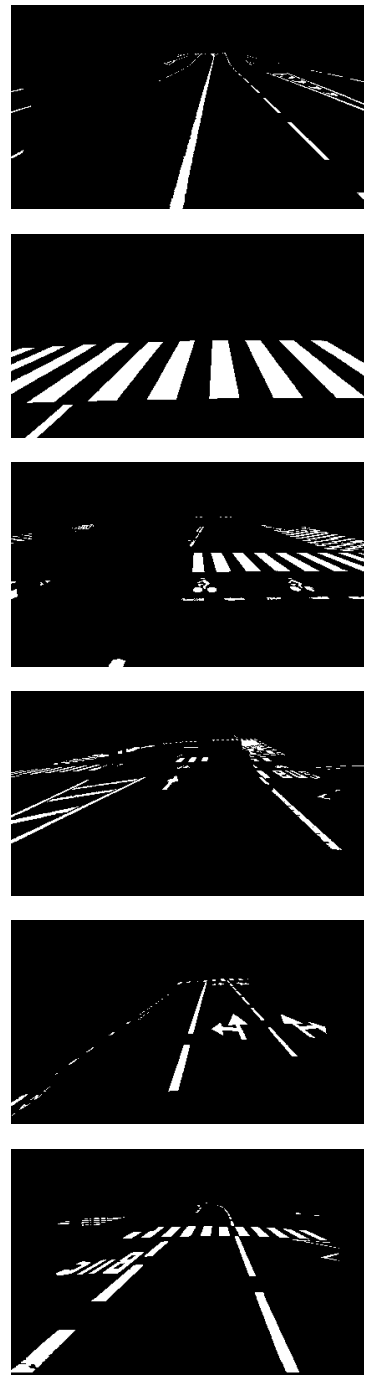
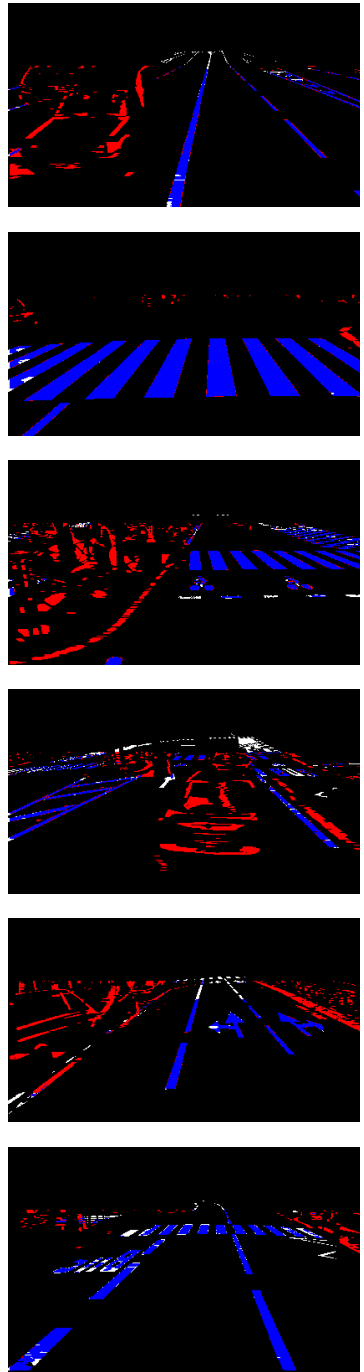
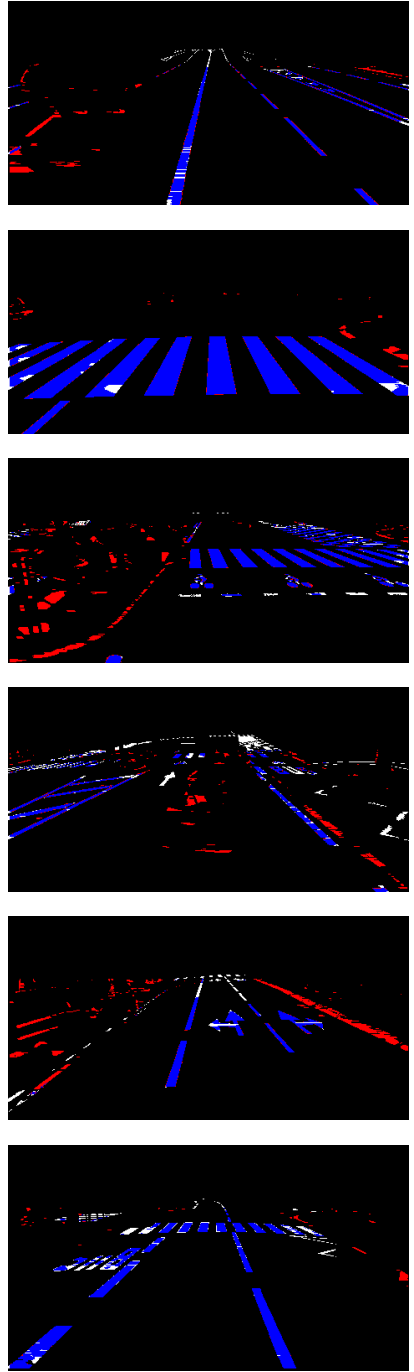

Fig. 8. First column original image. Second column ground truth (white : road markings, black : non-marking). Third column extractions results of the MLT algorithm in case of single-vision. Fourth column extractions results of the MLT algorithm in case of stereo-vision (red : False detections, blue : true detections)

obvious. The maximum of Dice Coefficient increases respectively by $5.29 \%, 5.20 \%$ and $8.82 \%$ for the MLT, SLT and LT extractors. Missed pixels by stereo selection are very likely due to variations of road plane (road slope, pitch angle...).

Considering that the extraction is the first step of the marking detection system, this loss of pixels does not appear dramatic as illustrated on the pedestrian crossing of Fig 8row 2. Indeed, it is only the border which is reduced a little, preserving the global shape of the marking and only introducing a small bias in its location. The main advantage of stereo selection is the decrease of FPR. It is particularly illustrated on Fig 8-row 4 where false alarms resulting from car extraction have been eliminated.

\section{CONCLUSIONS AND FUtURE WORK}

In this paper, we addressed the problem of extracting road marking elements in urban environment images. The problem is challenging, due to the presence of large special markings. 

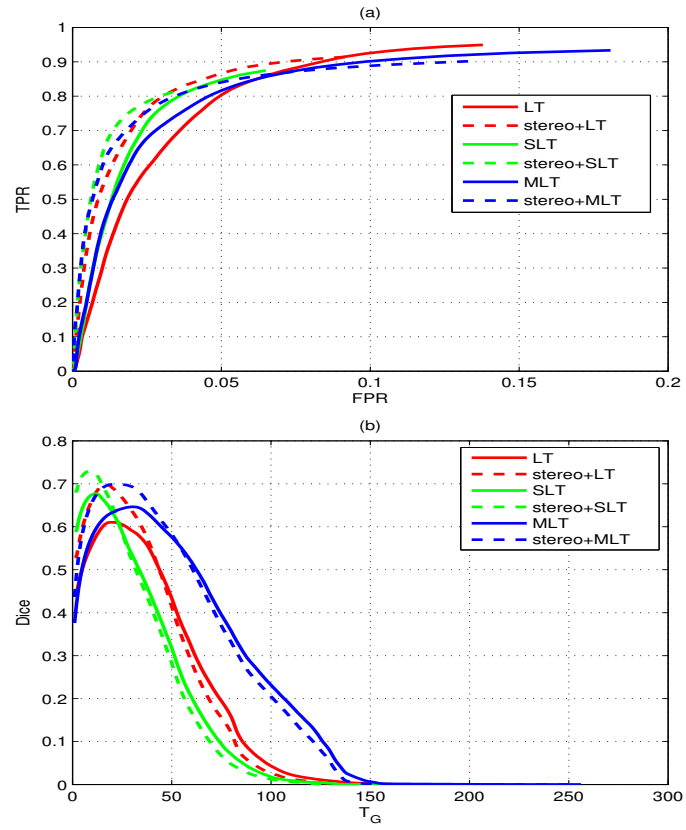

Fig. 9. Comparison between single-image and stereo versions of three extraction algorithms : Local Threshold $(L T)$, Symmetrical Local Threshold $(S L T)$ and Median Local Threshold $(M L T)$ with $43^{\text {rd }}$ percentile : Roc curves (a), Dice curves (b)

Compared to regular lane markings, special markings are in general more complex, have a more variable width, are larger and are sometimes denser. Moreover, there are many potential sources of false alarms and occlusions in urban images taken in the traffic. The algorithm we proposed in this paper is able to extract all kinds of road markings. The performances of its two versions (single-image and stereo) were evaluated and compared on a database of 47 images. We are planning to extend this database.

The results showed that the method is efficient to extract both road lane and special markings. Moreover, the use of a lower quantile ( $43^{\text {rd }}$ percentile) in the Median Local Threshold algorithm improves the pedestrian crossing and zebras extraction. Considering an extra stereo selection, the number of false alarms is strongly decreased, while the True Positive Rate is slightly reduced. As we already noticed, the decrease of TPR is observed in terms of pixelwise detection. It might not be dramatic for the application, provided the subsequent steps of the process are robust to missing pixels, as the method proposed in [16] for lane detection. This should be confirmed by further evaluations, including the overall process.

The fact that stereovision, when available, increases the performances of all extraction algorithms is an encouraging result, especially if we recall that the road plane was considered fixed to its calibrated position and orientation in our experiments. We believe that introducing road profile reconstruction [15] in the process will bring further improvements.

\section{ACKNOWLEDGMENTS}

The authors gratefully acknowledge the contribution of Philippe Nicolle and Bahman Soheilian in building the refe- rence database and of IGN in providing the stereo images. This work is partly funded by the ANR (French National Research Agency) within iTowns-MDCO project.

\section{REFERENCES}

[1] M. Aly. Real time detection of lane markers in urban streets. In IEEE Transactions on Intelligent Vehicles Symposium (IV'08), pages 7-12, 2008.

[2] N. Benmansour, R. Labayrade, D. Aubert, and S. Glaser. A model driven $3 \mathrm{~d}$ lane detection system using stereovision. In IEEE International Conference on Control Automation Robotic and Vision (ICARCV'08), Hanoi, Vietnam, 2008.

[3] A. Borkar, M. Hayes, and M. Smith. Lane detection and tracking using a layered approach. In Advanced Concepts for Intelligent Vision Systems, pages 474-484, 2009.

[4] J. Coughlan and H. Shen. A fast algorithm for finding crosswalks using figure-ground segmentation. In 2nd Workshop on Applications of Computer Vision, in conjunction with ECCV, volume 5, 2006.

[5] T. Fawcett. An introduction to ROC analysis. Pattern Recognition Letters, 27(9) :861-874, 2006.

[6] U. Franke, D. Gavrila, S. Gorzig, F. Lindner, F. Paetzold, and C. Wohler. Autonomous driving goes downtown. IEEE Intelligent Systems and their Applications, 13(6):40-48, 1998.

[7] U. Franke and I. Kutzbach. Fast stereo based object detection for stop and go traffic. In IEEE Intelligent Vehicles Symposium (IV'96), pages 339-344, 1996.

[8] A. Lopez, J. Serrat, C. Canero, and F. Lumbreras. Robust lane lines detection and quantitative assessment. In 3rd Iberian Conference on Pattern Recognition and Image Analysis, pages 274-281, Girona, Spain, 2007.

[9] J. McCall and M. Trivedi. Video based lane estimation and tracking for driver assistance : Survey, system, and evaluation. IEEE Transactions on Intelligent Transportation Systems, 7(1) :20-37, 2006.

[10] S. Nedevschi, R. Schmidt, T. Graf, R. Danescu, D. Frentiu, T. Marita, F. Oniga, and C. Pocol. 3D lane detection system based on stereovision. In Proceedings of IEEE Conference on Intelligent Transportation Systems (ITSC'04), pages 161-166, 2004.

[11] J. Ninot, J. Tarel, T. Gavrilovic, L. Smadja, and K. Heggarty. Amélioration de la reconnaissance des marquages routiers par l'optimisation d'algorithmes d'extraction. In Proceedings of colloque COGIST'09, St Quay Portrieux, France, 2009. http ://perso.lcpc.fr/tarel.jean-philippe/publis/cogist09.html.

[12] S. Perreault and P. Hebert. Median filtering in constant time. IEEE Transactions on Image Processing, 16(9) :2389-2394, september 2007.

[13] S. Se and M. Brady. Road feature detection and estimation. Machine Vision and Applications, 14(3) :157-165, 2003.

[14] B. Soheilian, N. Paparoditis, D. Boldo, and J. Rudant. Automatic $3 \mathrm{~d}$ extraction of rectangular roadmarks with centimeter accuracy from stereo-pairs of a ground-based mobile mapping system. In International Archives of the Photogrammetry, Remote Sensing and Spatial Information Sciences, pages 22-27, Padua, Italy, 2007.

[15] J. Tarel, S. Ieng, and P. Charbonnier. Accurate and robust image alignment for road profil reconstruction. In Proceedings of IEEE International Conference on Image Processing (ICIP'07), volume V, pages 365 368, San Antonio, Texas, USA, 2007. http ://perso.lcpc.fr/tarel.jeanphilippe/publis/icip07.html.

[16] J.-P. Tarel, S.-S. Ieng, and P. Charbonnier. Using robust estimation algorithms for tracking explicit curves. In Proceedings of European Conference on Computer Vision (ECCV'02), volume I, pages 492 507, Copenhagen, Denmark, 2002. http ://perso.lcpc.fr/tarel.jeanphilippe/publis/eccv02.html.

[17] M. Uddin and T. Shioyama. Robust zebra-crossing detection using bipolarity and projective invariant. In Proceedings of the Eighth International Symposium on Signal Processing and Its Applications, volume 2, 2005.

[18] T. Veit, J. P. Tarel, P. Nicolle, and P. Charbonnier. Evaluation of road marking feature extraction. In Proceedings of IEEE Conference on Intelligent Transportation Systems (ITSC'08), pages 174-181, Beijing, China, 2008. http ://perso.lcpc.fr/tarel.jean-philippe/publis/itsc08.html. 